\title{
Tandem high-dose therapy (HDT) for multiple myeloma: recombinant human erythropoietin therapy given between first and second HDT allows second peripheral blood stem cell transplantation without red blood cell transfusion
}

\author{
Frédéric Baron, Pascale Frère, Georges Fillet and Yves Beguin Department of Medicine, Division \\ of Haematology, University of Liège, Liège, Belgium
}

Received 15 April 2003; accepted for publication 30 May 2003

\begin{abstract}
Summary. We evaluated the ability of recombinant human erythropoietin (rHuEpo) therapy, given before high-dose therapy (HDT), to allow autologous peripheral blood stem cell transplantation (PBSCT) without red blood cell (RBC) transfusions. Eleven multiple myeloma patients underwent tandem HDT and autologous PBSC, receiving $500 \mathrm{U} / \mathrm{kg} /$ week rHuEpo from d 30 after initial transplant. Haemoglobin levels were $9.5 \pm 1 \cdot 1 \mathrm{~g} / \mathrm{dl}$ and $12.5 \pm 0.9 \mathrm{~g} / \mathrm{dl}$ at the first and second transplant respectively $(P<0 \cdot 001)$. RBC
\end{abstract}

transfusions were required for 10/11 patients for the first transplant versus $1 / 11$ for the second $(P<0 \cdot 001)$. To conclude, a short course of rHuEpo therapy before HDT facilitates the performance of an autologous transplant without RBC transfusions.

Keywords: autologous stem cell transplantation, tandem transplantation, myeloma, erythropoietin, recombinant erythropoietin.
Conventional red blood cell (RBC) transfusions are still associated with a low risk of a number of serious complications (Goodnough et al, 1999). It is now well established that recombinant human erythropoietin (rHuEpo) treatment raises haemoglobin $(\mathrm{Hb})$ levels, reduces the need for transfusions and improves quality of life in cancer-associated anaemia (Beguin, 2002). After haematopoietic stem cell transplantation (HSCT), several trials have administered very high doses of intravenous (i.v.) rHuEpo starting on d 1 and continuing for either 1-2 months or until erythroid engraftment. These trials have uniformly shown little (in allogeneic HSCT) or no (in autologous HSCT) clinical benefit (Link et al, 1994), with no reduction in transfusion needs observed. On the other hand, we have shown recently that rHuEpo was very efficient when started at least 1 month after an allogeneic transplant, at a time when erythropoietic marrow recovery is accomplished and endogenous erythropoietin (Epo) production is inappropriately low for the degree of anaemia (Baron et al, 2002a; Baron \& Beguin, 2003). However, this approach would not impact on

Correspondence: Yves Beguin, University of Liège, Department of Haematology, CHU Sart Tilman, 4000 Liège, Belgium. E-mail: yves.beguin@chu.ulg.ac.be transfusion requirements when they are most prominent, i.e. in the first month after transplantation.

Tandem autologous HSCT in first-line therapy has been shown to be an optimal treatment option for multiple myeloma (Attal et al, 2002). The aim of this study was to investigate whether rHuEpo treatment starting $30 \mathrm{~d}$ after the first transplant could increase $\mathrm{Hb}$ levels sufficiently to abrogate the need for RBC transfusion after the second HSCT.

\section{PATIENTS AND METHODS}

We developed a protocol of rHuEpo therapy in multiple myeloma patients in first-line treatment undergoing tandem autologous peripheral blood stem cell transplantation (PBSCT), with the aim of avoiding RBC transfusions in the second HSCT procedure. RhuEpo was not given before the first transplant, so that patients served as their own internal controls. Eleven patients, five males and six females, aged 44-64 (median 58) years, were included. The collection and infusion of PBSC was carried out as described previously (Andre et al, 2003). The percentage of bone marrow plasmocytes was $2 \pm 4 \%$ and $1 \pm 1 \%$ before the first and second transplants respectively. Before the first transplant, polymorphonuclear leucocytes $(\mathrm{PMN})$ and platelet counts were $2 \cdot 79 \pm 1 \cdot 35$ and $271 \pm 144 \times 10^{9} / 1$ respectively. The 
Fig 1. (A) Change in sTfR between d 0 and d 21 after the start of rHuEpo therapy. (B) Hb level on d 30 and 100 after PBSCT in patients treated with rHuEpo $(n=11)$ or in historical controls $(n=11)$. NS, not significant. (C) Hb evolution after the two PBSCT procedures. (D) RBC transfusions in the two PBSCT procedures.

conditioning regimen was $200 \mathrm{mg} / \mathrm{m}^{2}$ melphalan for both transplants. Patients received a mean of $13 \cdot 8 \pm 10 \cdot 3$ and $15 \cdot 5 \pm 10 \cdot 3 \mathrm{CD} 34^{+}$cells $/ \mathrm{kg}$ as first and second transplant, respectively, representing a non-significant difference, and were treated with granulocyte colony-stimulating factor (G-CSF) after transplantation. Single-donor platelet transfusions were given if platelet counts decreased below $15 \times 10^{9} /$ l. The trigger for RBC transfusions was an $\mathrm{Hb}$ level of $8 \mathrm{~g} / \mathrm{dl}$ or below.

Patients were scheduled to start subcutaneous (s.c.) rHuEpo on d $30 \pm 3$ after the first PBSCT at a dose of $500 \mathrm{U} / \mathrm{kg} /$ week (standard dose for cancer anaemia) with the aim of achieving $\mathrm{Hb}$ levels of $13 \mathrm{~g} / \mathrm{dl}$. Once that target was achieved, the dose was reduced, in order to use the lowest dose capable of maintaining the $\mathrm{Hb}$ between 12 and $14 \mathrm{~g} / \mathrm{dl}$. As the second HSCT procedure was performed $112 \pm 18 \mathrm{~d}$ after the first, patients were treated for an average of 12 weeks. Patients with functional iron deficiency (defined by transferrin saturation $<20 \%$ ) received i.v. saccharose-iron (Venofer $\AA$ ) at a dose of $200 \mathrm{mg} /$ week for 3 weeks.

Complete blood counts, reticulocytes, serum soluble transferrin receptor (sTfR) (a quantitative measure of total erythropoietic activity) (R'Zik \& Beguin, 2001), serum Epo and the observed-to-predicted $(\mathrm{O} / \mathrm{P})$ ratio were measured as reported previously (Baron et al, 2002a,b; Beguin et al, 1993).

Unpaired and paired Student's t-tests were used to compare biological variables in two groups or to compare baseline values with later measurements in the same group of patients. Welsh's correction was used in cases of unequal variance. The number of patients requiring transfusions was compared using Fisher's exact test.

\section{RESULTS}

Efficacy of rHuEpo therapy after the first transplant

The O/P Epo ratio was $0.85 \pm 0 \cdot 11$ on $\mathrm{d} 30$ after the first PBSCT, showing relative endogenous Epo deficiency. RhuEpo therapy was started on d $31 \pm 4$. Erythropoiesis, as assessed by sTfR, increased very quickly from $6577 \pm$ $2539 \mu \mathrm{g} / \mathrm{l}$ at baseline to $10206 \pm 2233 \mu \mathrm{g} / \mathrm{l}(P<0 \cdot 0001)$ 3 weeks later (Fig 1A). Reticulocytes increased from $66 \cdot 2 \pm 25 \cdot 7 \times 10^{9} / 1$ to $107 \cdot 7 \pm 50 \cdot 1 \times 10^{9} / 1$ during the same period $(P=0.007)$. The median time to an $\mathrm{Hb}$ increment $\geq 2 \mathrm{~g} / \mathrm{dl}$ was 4 weeks, and eight out of 11 patients achieved an $\mathrm{Hb}$ of $13 \mathrm{~g} / \mathrm{dl}$ after a median of 8 weeks. The $\mathrm{Hb}$ level increased from $9.6 \pm 1.0 \mathrm{~g} / \mathrm{dl}$ at baseline to $13.9 \pm 1.4 \mathrm{~g} / \mathrm{dl}$ on $\mathrm{d} 100(P<0.001)$. There was no correlation with the $\mathrm{CD} 34^{+}$cell dose received. In

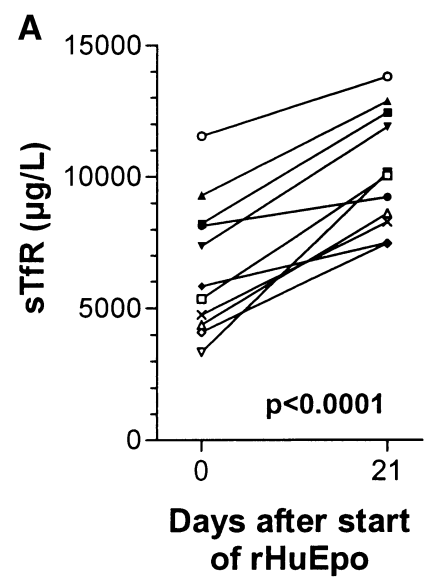

B
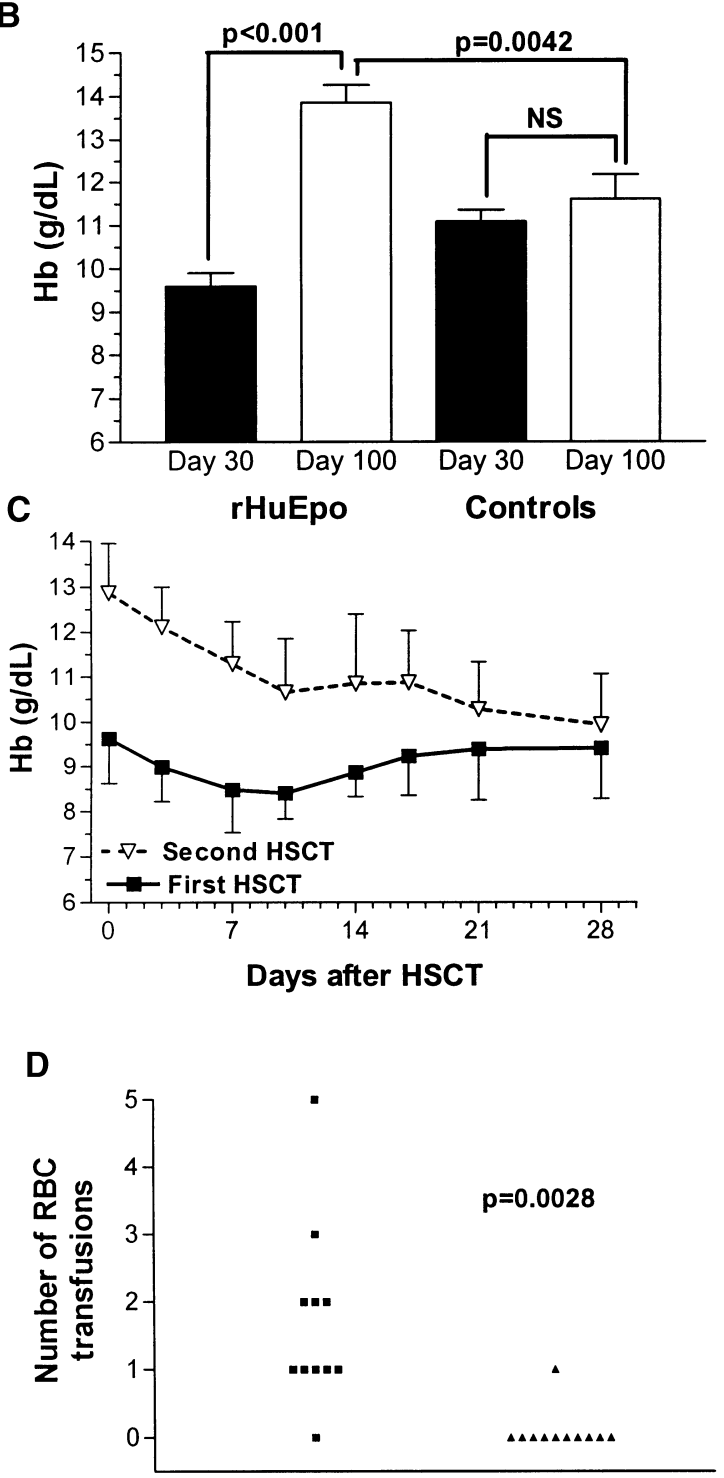

First PBSCT Second PBSCT 
comparison, in an historical group of 11 transplanted myeloma patients, $\mathrm{Hb}$ increased from $11.1 \pm 0.9$ to $11.6 \pm 1.9 \mathrm{~g} / \mathrm{dl}$ in the same interval $(P=0.0042$ for comparison with d $100 \mathrm{Hb}$ in rHuEpo-treated recipients) (Fig 1B).

Comparison of the two transplant procedures

The $\mathrm{Hb}$ level was $9 \cdot 5 \pm 1 \cdot 1 \mathrm{~g} / \mathrm{dl}$ at first transplant versus $12.5 \pm 0.9 \mathrm{~g} / \mathrm{dl}$ at second transplant. $\mathrm{Hb}$ values after the second transplant remained higher throughout the first 2 weeks but reached identical levels on d 28 (Fig 1C). In both transplants, erythropoietic activity decreased after the conditioning regimen, but recovered progressively after $\mathrm{d} 7$. These findings demonstrate that $\mathrm{Hb}$ differences between the two PBSCT only resulted from higher $\mathrm{Hb}$ values achieved before PBSCT, and not increased erythropoiesis after the second PBSCT.

\section{Transfusion needs}

Ten out of 11 patients required RBC transfusions for the first PBSCT, versus one out of 11 for the second transplant $(P<0.001)$ (Fig 1D). RBC and platelet requirements were $1 \cdot 7 \pm 1 \cdot 3$ and $1 \cdot 0 \pm 1 \cdot 1$ for the first procedure versus $0 \cdot 1 \pm 0 \cdot 3(P=0 \cdot 003)$ and $0 \cdot 5 \pm 0 \cdot 7(\mathrm{NS})$ for the second procedure respectively.

\section{DISCUSSION}

Our data demonstrate first the remarkable efficacy of rHuEpo therapy when started $30 \mathrm{~d}$ after an autologous HSCT, when endogenous Epo production becomes inappropriately low for the degree of anaemia (Beguin et al, 1998), achieving a normal $\mathrm{Hb}$ level on d 100, just before the second transplant. In addition, our data demonstrate that the higher $\mathrm{Hb}$ levels obtained by rHuEpo therapy after the first transplant permitted the carrying out of the second HSCT procedure without RBC transfusions in $>90 \%$ of the patients. Ponchio et al (2000) have reported previously, in 10 breast cancer patients, that $\mathrm{RBC}$ transfusion requirements can be decreased by rHuEpo therapy started after PBSC collection. However, in that study, patients were compared with historical controls, whereas in our study, patients served as their own internal controls, receiving exactly the same treatment schedule with or without rHuEpo before autologous transplantation. In addition, whereas it was already known that cancer patients on chemotherapy could respond to rHuEpo, we show here for the first time that rHuEpo is at least as efficient when given shortly after recovery from first autologous HSCT.

In conclusion, in tandem autologous PBSCT for multiple myeloma, rHuEpo therapy started $30 \mathrm{~d}$ after the first PBSCT permitted performance of the second PBSCT without RBC transfusion.

\section{ACKNOWLEDGMENT}

Frédéric Baron is Research Assistant and Yves Beguin Research Director of the National Fund for Scientific Research (FNRS, Belgium). This work was supported in part by grants from the FNRS.

\section{REFERENCES}

Andre, M., Baudoux, E., Bron, D., Canon, J.L., D’Hondt, V., Fassotte, M.F., D’Hondt, L., Fillet, G., Humblet, Y., Jerusalem, G., Vermeulen, P., Symann, M. \& Beguin, Y. (2003) Phase III randomized study comparing 5 or 10 microg per kg per day of filgrastim for mobilization of peripheral blood progenitor cells with chemotherapy, followed by intensification and autologous transplantation in patients with nonmyeloid malignancies. Transfusion, 43, 50-57.

Attal, M., Harousseau, J.L., Facon, T., Guilhot, F. \& Doyen, C. (2002) Double autologous transplantation improves survival of multiple myeloma patients: final analysis of a prospective randomized study of the intergroupe francophone du myelome. Blood, 100, 7a.

Baron, F. \& Beguin, Y. (2003) Once weekly recombinant human erythropoietin therapy is very efficient after allogeneic peripheral blood stem cell transplantation (alloPBSCT) when started soon after engraftment. Haematologica, 88, 718-720.

Baron, F., Sautois, B., Baudoux, E., Matus, G., Fillet, G. \& Beguin, Y. (2002a) Optimization of recombinant human erythropoietin therapy after allogeneic hematopoietic stem cell transplantation. Experimental Hematology, 30, 546-554.

Baron, F., Fillet, G. \& Beguin, Y. (2002b) Erythropoiesis after nonmyeloablative stem-cell transplantation is not impaired by inadequate erythropoietin production as observed after conventional allogeneic transplantation. Transplantation, 74, 1692-1696.

Beguin, Y. (2002) Prediction of response and other improvements on the limitations of recombinant human erythropoietin therapy in anemic cancer patients. Haematologica, 87, 1209-1221.

Beguin, Y., Clemons, G.K., Pootrakul, P. \& Fillet, G. (1993) Quantitative assessment of erythropoiesis and functional classification of anemia based on measurements of serum transferrin receptor and erythropoietin. Blood, 81, 1067-1076.

Beguin, Y., Baron, F. \& Fillet, G. (1998) Influence of marrow activity on serum erythropoietin levels after autologous hematopoietic stem cell transplantation. Haematologica, 83, 1076-1081.

Goodnough, L.T., Brecher, M.E., Kanter, M.H. \& AuBuchon, J.P. (1999) Transfusion medicine. First of two parts - blood transfusion. New England Journal of Medicine, 340, 438-447.

Link, H., Boogaerts, M.A., Fauser, A.A., Slavin, S., Reiffers, J., Gorin, N.C., Carella, A.M., Mandelli, F., Burdach, S. \& Ferrant, A. (1994) A controlled trial of recombinant human erythropoietin after bone marrow transplantation. Blood, 84, 3327-3335.

Ponchio, L., Zambelli, A., De Stefano, A., Robustelli Della Cuna, F.S., Perotti, C. \& Pedrazzoli, P. (2000) Transfusion requirement can be abolished by epoietin-a and autologous platelet predeposit in patients receiving high dose chemotherapy with stem cell support. Haematologica, 85, 219-220.

R'Zik, S. \& Beguin, Y. (2001) Serum soluble transferrin receptor concentration is an accurate estimate of the mass of tissue receptors. Experimental Hematology, 29, 677-685. 